\title{
Téoros
}

Revue de recherche en tourisme

\section{La valeur touristique et économique des paysages}

\section{Michel Zins et Julie Jacques}

Volume 18, numéro 1, printemps 1999

Les jardins du tourisme

URI : https://id.erudit.org/iderudit/1072300ar

DOI : https://doi.org/10.7202/1072300ar

Aller au sommaire du numéro

Éditeur(s)

Université du Québec à Montréal

ISSN

0712-8657 (imprimé)

1923-2705 (numérique)

Découvrir la revue

Citer cet article

Zins, M. \& Jacques, J. (1999). La valeur touristique et économique des paysages. Téoros, 18(1), 48-51. https://doi.org/10.7202/1072300ar d'utilisation que vous pouvez consulter en ligne.

https://apropos.erudit.org/fr/usagers/politique-dutilisation/ 


\section{LA VALEUR TOURISTIQUE ET ÉCONOMIQUE DES PAYSAGES}

\section{Michel Zins et Julie Jacques}

La valeur touristique - et donc la valeur économique des paysages - ne fait plus de doute même si aucune méthode universelle d'évaluation de cette valeur n'a encore été développée.

Les paysages sont en tête de liste des raisons qui motivent le choix d'une destination de voyagé. Le Canada bénéficie de son positionnement fort quant à la qualité des paysages. Par ailleurs, l'unicité et la qualité des paysages déterminent le choix des destinations pour la pratique de plus en plus d'activités touristiques spécifiques comme le vélo, le quad, la motoneige et le tourisme d'aventure.

Fïnalement, au-delà des retombées économiques de la fréquentation et des dépenses directes qu'ils génèrent par leur pouvoir d'atraction et d'émerveillement des touristes, les paysages représentent également une importante valeur comme e marque de commerce sour une région qui peut s'identifier à ses paysages et être reconnue pour leur beaute en leur unicite.

La valeur des paysages est enfin confirmée par les énormes investissements requis pour combler l'absence de paysages distinctifs ou pour rétablir la qualité de certains paysages et environnements qui ont été saccagés. C'est une valeur économique et un capital que toute destination doit préserver et metire en valeur. Plusieurs régions du Québec particulièrement, offrent des paysages uniques de premier ordre.

Depuis plusieurs années le tourisme est finalement perçu pour ce qu'il est, une des plus grandes industries au monde, le plus important employeur, un des secteurs qui connaît la plus forte croissance, un vecteur de développement régional et social.

En tourisme, la valeur économique directe résulté d'un achalandage de touristes, comportant des nuitées et générant des recettes touristiques aux chapitres de l'hébergement, de la restauration, des attraits, des activités et services, ainsi que du transport dont peuvent bénéficier une ville, une région, une entreprise du fait de leur capacité d'attirer des touristes.

De multiples raisons expliquent l'attrait d'une région touristique. Dans le groupe de tête figurent les paysages, le plus souvent naturels, mais aussi bâtis.

Quelle est la valeur économique des paysages pour l'industrie touristique ? On n'a pas encore développé aucun modèle d'estimation qui permet d'évaluer de façon précise et universelle la valeur économique des paysages d'une localité, d'une région, d'un territoire. Et il n'est pas sûr qu'un tel outil puisse être développé pour évaluer toutes les situations. Il n'est pas forcément utile non plus.

Cependant, les éléments sous-jacents au choix d'une destination touristique plutôt qu' une autre, ainsi que les facteurs qui expliquent l'attraction d"une destination touristique, représentent autant d'indicateurs de la valeur touristique déjả connus et mesurés et, conséquemment, de la valeur économique des paysages. Les intentions et les choix de destinations touristiques se forment à partir des perceptions que les touristes ont des motivations qui les animent et des expériences touristiques qu'ils recherchent.

Par ailleurs, l'attraction d'une destination touristique s'établit sur la base de ses caractéristiques propres et de son unicité qui lui confèrent un avantage concurrentiel distinctif par rapport à une autre région ou à une autre destination. L'aspect * scénique $*$ du paysage ( $*$ scenery $*$ en anglais) attire et enveloppe l'expérience vécue par le touriste. Il peut se véhiculer alors de façon unique et reconnaissable dans des reportages, des cartes postales, de la publicité.

Indêniablement, le paysage, de par son caractère généralement unique, est le visage d'une localité, d'une région, d'un

\begin{tabular}{|c|c|c|c|}
\hline \multicolumn{4}{|c|}{ TABLEAU 1} \\
\hline * Les fjords de Norvège & - Les lacs suisses & - La Corse & - Le Pérou \\
\hline - La campagne irlandaise & - Les Rocheuses & - La Californie & - Rio \\
\hline * Les Alpes & - Le Sahara & - Le Grand Canyon & - San Francisco \\
\hline
\end{tabular}


territoire. Le paysage est une richesse de premier ordre : la principale valeur intrinsèque, le patrimoine, l'équité d'une région. Ainsi, le paysage, de par son caractère distinctif et unique, est un atout, un avan= tage concurrentiel, sur lequel une région peut miser et se positionner pour attirer les touristes.

Les paysages sont la force attractive de bien des destinations par ailleurs fort différentes (voir tableau 1).

Dans toutes ces destinations, les touristes souvent ne connaissent rien d'autre qu une image de carte postale les représentant.

Cependant, tous les paysages et toutes les destinations n'ont pas la mểme force attractive.

\begin{tabular}{|l|}
\multicolumn{1}{c|}{ TABLEAU 2} \\
Les voyageurs français \\
Segment culture et nature \\
$(13 \%$ du marché total $)$
\end{tabular}

Source : Market Facts of Canada Limited, LAs marches do voyage ơ agrément en Amérique du Nond : France, Tourisme Canada, Novembre 1989, 158 p.

\section{TABLEAU 4}

Les voyageurs japonais Segment culture et nature (23\% du marché total)

- Groupes culturels différents

- Petites villes et petits villages d'intérét

- Sites et édifices historiques et archéologiques

- Étendues sauvages

- Faune et oiseaux

- Marche et escalade

- Logements et restaurants bon marche

Source = Market Facts of Canada Limited, Les marchos du voyage d'agrément en Amérique du Nord : Japon, Tourisme Canada, Novembre 1989, 151 p.

\section{LE PAYSAGE COMME PATRIMOINE}

C'est ainsi que, pour compenser le caractère plutốt quelconque d'un paysage ou pour valoriser certains atouts d'un paysage qui seraient naturellement peu perceptibles ou peu accessibles, certaines régions doivent procéder à des investissements considerables pour se positionner sur l'echiquier touristique.

Que l'on pense seulement à la région de Kissimmee/ Orlando avec son Disney World bâti au milieu d'une immense zone marécageuse, à Toronto avec son imposant Waterfront, ses attraits et ses expositions, à Philadelphie et sa zone historique, à Boston et son port qui investissent abondamment pour compenser un manque de

\begin{tabular}{|c|}
\hline \multicolumn{1}{c|}{ TABLEAU 3} \\
Les voyageurs britanniques \\
Segment culture et nature \\
$(16 \%$ du marché total)
\end{tabular}

Source : Market Facta of Cinada Limited. Les marches du vorage d"agriment en Amdrigue du Nord : Royaume-Uni, Tourisme Canada, Nowembre 1989, 158 p.

\section{TABLEAU 5}

\section{Les voyageurs allemands Segment culture et nature (17 $\%$ du marché total)}

- Groupes culturels différents

- Faune et oiseaux

- Régions sauvages

- Parcs nationaux et forêts

- Régions montagneuses

- Paysages spectaculaires

- Marche et escalade

Source : Market Facts of Caneda Limited, Les marchés du voyage d'agriment en Amenique du Nord : Allemagne de f'Ouest. Tourisme Canada, Novambre 1989, $158 \mathrm{p}$. paysages naturels et pour reconstituer des paysagés saccagés ou négligés.

Si l'on compare ces régions à San Francisco, à Québec, ville du patrimoine mondial, aux Alpes, il est clair que les investissements compensatoires constituent une mesure de la valeur économique du paysage et du capital qu'ils représentent et, surtout, du coût requis pour compenser leur manque ou pour remédier à leur dégradation.

\section{LE PAYSAGE COMME IMAGE DE MARQUE}

Les motifs de choix d'une destination touristique et l'image qu'en ont les touristes constituent une deuxième mesure ou, du moins, un deuxième indicateur de la valeur des paysages.

En effet, pour de nombreuses destinations, les raisons déterminantes du choix des touristes peuvent dépendre d'un attrait spécifique ou d'un micro-attrait tel qu'une montagne, un lac / un plan d'eau, une forêt, une île. Mais, en réalité, ce que les touristes visualisent c'est moins les attraits individuels et d'avantage les paysages dans leur ensemble : un panorama, un paysage $\mathrm{di}-$ versifié, un paysage d'une grande beauté. de grands espaces, un paysage rural, un village typique, un patrimoine urbain exceptionnel et unique... A ce chapitre, les motivations de voyage des clientèles géographiques sont fort évocatrices.

\section{LES PAYSAGES COMME MOTIF DE VOYAGE}

Dans les motifs de voyages des touristes canadiens, les panoramas exceptionnels figurent parmi les principaux déterminants de leur choix de destination de voyages (les voyages au Canada : $73 \%$, les voyages aux Etats-Unis : $72 \%$, les voyages dans d'autres pays : $89 \%$ )'.

Par ailleurs, les touristes américains qui voyagent au Canada ont, comme principales motivations de voyage, l'experience touristique globale et la visite de grandes villes. Parmi ce qui les attire le plus au Canada, on note l'exotisme, les panoramas, le plein air.

Quant aux touristes d'outre-mer, ce sont les grands espaces qui les attirent. Les 
Français, aussi, fréquentent d'abord les pares nationaux et les forets ${ }^{2}$ (voir tableau 2).

Les Britanniques aiment admirer les pay= sages et, pour eux, la qualité de l'environnement local devient un facteur de plus en plus important dans le choix d'une destination. Ils sont attirés au Canada pour la beauté majestueuse de la nature et les paysages pittoresques ${ }^{3}$ (voir tableau 3 ).

Les Japonais participent à des activités de plein air, admirent les paysages et visitent les parcs nationaux, les forêts ou les régions montagneuses ${ }^{4}$ (voir tableau 4 ).

Les Allemands axent beaucoup leurs voyages sur la combinaison culture nature. Ils s'intéressent au Canada pour sa beauté naturelle et ses grands espaces. Ils admirent les paysages, visitent les pares nationaux et les forêts, visitent des régions sauvages $^{5}$ (voir tableau 5 ).

A cet égard, Charlevoix est un bon exemple. Une étude conduite en 1997 révẻle que les clientèles y viennent pour la découverte des paysages - c'est là l'expérience recherchée qui domine. Les paysages sont aussi la principale image que les clientèles actuelles et potentielles ont de la région. Par ailleurs, les paysages sont également à la source des intentions de retour autant que des intentions de premiêre visite (voir tableau 6).

Si l'on considère l'importance des paysages dans les motivations de visite de certaines régions,il est évident que chacune de ces régions, sans ses paysages uniques et distinctifs, ne bénéficierait pas de la performance actuelle au chapitre du volune de touristes (Vancouver : 8000000 , Québec : 3800000 , Charlevoix : 700000 ) ni au chapitre des recettes touristiques (Vancouver : 2460 millions de dollars, Québec : 813 millions de dollars, Charlevoix : 90 millions de dollars).

Meme si elle est difficilement mesurable, la valeur touristique et économique des paysages est indéniable.

\section{LE PAYSAGE COMME VALORISATION}

Par ailleurs, le paysage apporte une valorisation complémentaire à certaines activités récréotouristiques auxquelles il sert de toile de fond et souvent de choix de la destination où la pratique de l'activité va se tenir.

C'est ainsi que des activités de plein air et d'aventure douce, ainsi que des activités spécialisées telles que la motoneige, le quad / VTT, le vélo qui, traditionnellement, étaient des activités de loisirs récréatifs, développent un potentiel touristique croissant en prenant appui sur les paysages.

Passant de statut de $\alpha$ loisirs sportifs $\$$ au statut de « moyen de découverte de régions et de territoires , ces activités s"inscrivent dans la tendance forte du tourisme actif.

La motoneige est une activité qui a pris. depuis quelques années, un virage touristique important en raison de l'exotisme et de l'aventure qu'elle porte en elle et de la curiosité qu'clle suscite. Elle est aussi devenue un moyen privilégié de découverte de l'hiver au Québec. Aussi, de plus en plus, des sentiers biens aménagés et entretenus ne suffisent plus aux touristes motoneigistes traditionnels (Québec, ÉtatsUnis, Ontario). La possibilité de traverser

\begin{tabular}{|c|c|c|}
\hline \multicolumn{3}{|c|}{ TABLEAU 6} \\
\hline $\begin{array}{l}\text { IMAges PERÇUES ET Associées } \\
\text { Ȧ LA RÉGION DE CHARLFVOIX }\end{array}$ & $\begin{array}{l}\text { CUENTELES ACTUELLES } \\
\text { ( } \% \text { DES MENTONS) }\end{array}$ & $\begin{array}{l}\text { Clientèles potentielues } \\
\text { ( } \% \text { des mentions) }\end{array}$ \\
\hline $\begin{array}{l}\text { - Grand air / espace / nature / forêts / } \\
\text { verdure / campagne }\end{array}$ & $35 \%$ & $21 \%$ \\
\hline - Paysage / beauté / panorama & $31 \%$ & $23 \%$ \\
\hline * Montagne & $21 \%$ & $14 \%$ \\
\hline - Fleuve / mer & $20 \%$ & $13 \%$ \\
\hline - Beauté des villages & $7 \%$ & \\
\hline
\end{tabular}

Source : Zins Beauchesne et associes, Etude des perceptions des clientales a repand de fó rogion touristique de Charlo voix, Association touristiqus de Charlevolx, 3 décembre 1997.

\begin{tabular}{|c|c|}
\hline \multicolumn{2}{|r|}{ TABLEAU 7} \\
\hline $\begin{array}{l}\text { AsPeCtS LES PLUS APPEÉCIÉS EN VOYAGE } \\
\text { DE MOTONEIGE }\end{array}$ & $\begin{array}{l}\text { ASPECTS IMPORTANTS RECHERCHÉS LORS DE VOYAGES } \\
\text { DE MONTAGNE }\end{array}$ \\
\hline $\begin{array}{l}\text { - Visiter de nouvelles régions } \\
\text { - Prendre de l'air } \\
\text { - Bien manger } \\
\text { - Passer de belles soirée }\end{array}$ & $\begin{array}{l}\text { - Des paysages agréables } \\
\text { - Des sentiers bien aménagés et entretenus } \\
\text { - Une signalisation adéquate } \\
\text { - Des services offerts le long des sentiers } \\
\text { - La disponibilité des cartes }\end{array}$ \\
\hline
\end{tabular}

Source : Zins Bnaucheane ot assocites, Enquête supres des membres nord-améritains de la Federotion des olubs de motoneigistes du Quebec. Federation des clubs de motoneigistes du Quebec, novernbre 1996

\section{TABLEAU 8}

\begin{tabular}{|c|c|}
\hline $\begin{array}{l}\text { AsPECTS LES PLUS AMMES DANS LES RÉGIONS VISITÉES } \\
\text { DANS LE CADRE DE VOYAGES DE MOTONEIGE }\end{array}$ & $\begin{array}{l}\text { ACTIVITÉS SUSCITANT LE PLUS D'INTÉPÉt } \\
\text { A Y ACCÉDER EN MOTONEIGE }\end{array}$ \\
\hline $\begin{array}{l}\text { - Les paysages / montagnes / nature / laćs } \\
\text { * Les sentiers / bonnes conditions } \\
\text { - Les gens / hospitalité / service } \\
\text { * L'hébergement / hôtellerie / relais } \\
\text { - La neige } \\
\text { + Les restaurants / la nourriture }\end{array}$ & $\begin{array}{l}\text { - Les sites panoramiques } \\
\text { - Les parcs naturels } \\
\text { - Des chutes } \\
\text { - Les restaurants gastronomiques }\end{array}$ \\
\hline
\end{tabular}

Souros : Zins Beauchesne et essociás, Evalustion des impsots économiques directs de la pratique fouristique de la motoneige au Québec, Federation dés clubs de motoneigistes du Québec, 20 nowembre 1996. 
ou d'accéder à des paysages uniques est le principal attrait $\mathrm{d}^{*}$ un circuit ou $\mathrm{d}^{+}$une destination $^{\top}$ (voir tableaux 7 et 8 ).

Quant aux Europeens, les motivations motoneige sont explicites : contact avec l'activité motoneige, prendre de $\mathrm{I}^{+} \mathrm{air}$, grands espaces, aventure, paysages (montagnes, lacs, animaux, ...), évasion, découvrir la nature ${ }^{8}$.

L'orientation « plaisir de la nature * et a decouvrir le Québec et ses paysages . $s$ accentue. La pratique de la motoneige évolue vers une expétience véritablement touristique de decouverte et d'appréciation du Québec hivernal, rural, de son environnement naturel et de ses paysages.

La motoneige génere des recettes de $413 \mathrm{M} \$$ dont $48 \%$ se rapportent à des voyages de motoneige ( $200 \mathrm{M} \$$ ) par des touristes du Québec $(67 \%)$, d'outre-mer (18\%) et des Etats-Unis (15\%) largement motivés par les paysages?

A l'instar de la motoneige, les activités. quad et vélo sont en plein développenent et promettent des potentiels touristiques. incontestables.

Les quadistes et leurs motivations évoluent. Ils recherchent des sentiers bien aménagés, une signalisation adéquate et l'aceès à des paysages agréables. Ils accordent une grande importance aux aspects suivants : sentiers biens aménagés ( $82 \%$ ), signalisation adéquate $(76 \%)$, paysages agréables $(69 \%)$, decouverte de nouvelles régions (41\%). Et leur's comportements touristiques se précisent au Québec: 91540 quadistes et $2300000 \mathrm{jrs} / \mathrm{an}$ (excursions), dont 40300 toutistes pour 1200000 jrs / an (voyages). Le quad est une activité économique de $163 \mathrm{M} \$$, grandement motivée par les paysages ${ }^{10}$.

L'activité velo évolue de la même façon, de telle sorte que deux segments de marché se dégagent : les cyclotouristes qui effectuent tout le trajet à vélo et dont le vélo est l'objectif premier du voyage; les touristes qui font la route en voiture, afin de se rendre dans une région ou sur un circuit où ils pourront faire du velo pendant quelques heures par jour pour profiter des paysages et accéder aux attraits touristiques. Pour ceux-ci la decouverte de la région est l'objectif premier et le vélo est le moyen pour y parvenir".
Parmi les principaux critères de choix d'une destination pour la pratique du toulrisme cycliste, on note, en ordre d'importance, les suivants: la beauté de la région visitée (paysages), la densité de la circulation routière, la culture et l'originalité de la région, les choses à faire ou à voif le jour, le prix, la disponibilité de cartes cyclistes. Peu importe la destination choisie et le degré de difficulté recherché, la beauté des paysages et les différents services / attraits restent une priorité pour la plupart des cyclistes ${ }^{12}$.

La valeur économique de l'activité touristique vélo se traduit par une injection directe de quelque $60 \$$ par nuitée (comparé à quelque $5 \$$ à 10 \$ par jour pour l'activité récréative), aussi induite en grande partie par l'accès à des paysages ${ }^{13}$.

Quant aux adeptes de tourisme $\mathrm{d}^{+}$aventure ayant fait un voyage au Quebec, ils n'hesitent pas à qualifier d'exceptionnelles, les activités d'observation de la nature et des paysages (ex. : rapides, chutes, lacs). Les grands espaces accessibles constituent un des principaux élements de satisfaction des voyages d'aventure au Québec. D'ailleurs, les attentes préalables à l'égard de la destination québecoise se rapportent au caractère extraordinaire des paysages ${ }^{14}$.

En conclusion, les paysages génèrent:

Une valeur touristique et économique, sil'on considere les investissements compensatoires requis...

Une valeur atraction principale, et donc de retombees economiques directes, dans les régions ou ils sont distinctifs et relativement wiques...

Une valear complémentaine d'attraction pour certaines activites lourishiques specialisees...

Madame Julie Jacques, B.A.A., M.B.A., assoclée el directrice du secteur tourisme de Zins Beanchesne et associés, est consultante en analyse et planification stratégique marketing et charge du cours Marketing towristique du Certificat en gestion du développement torristique de l'Universite Laval.

Monsieur Michel Zins, Ph. D., professew de marketing a l'Universite Laval et president de Zins Beauchesne et associes, est expert en analyse et planification strategique dans le sectear touristique et cumule vingt ans d'activites=consell et de recherche en towrme an service de plasieurs organisations prives ou publiques doni Tourisme Quebec, Parcs Canada, de nombreuses associations touristiques

et offices du towrisme.

\section{NOTES}

1 Coopers \& Lybrand (1996), Eude de recherche sur le marche interieur du tourisme. Canada rapport principal, Commission canadienne du Tourisme, $104 \mathrm{p}$.

2 Market Facts of Canada Limited (1989), Les marches du voyage d agrement on Amerique du Nord : France, Tourisme Canada, novembre, $158 \mathrm{p}$.

3 Commission canadienne du Tourisme (1997), Communique, édition speciale, janvier, $12 \mathrm{p}$.

4 Ihid.

5 Ibid.

6 Zins Beauchesne et associés (1997), Eude des percepions des clienteles a l'egard de la region touristique de Charlevoix, Asso= ciation touristique de Charlevoix, 3 décembre.

7 Zins Beauchesne et associés (1996), Enquêté aupres des menbres nord-americains de la Fédération des clubs de motoneigistes du Québec, Fédération des clubs de motoneigistes du Québec, novembre.

8 SOM Recherche et sondage (1998), La motoneige au Quebec vue par les Européens, Tourisme Quebec.

9 Zins Beauchesne et associés ( 1996 ), Evaluation des impacts économiques directs de la pratique fouristique de la motonelge au Quebec, Federation des clubs de motoneigistes du Québec, 20 novembre.

10 Pluram et Zins Beauchesne et associés (1996), Elrde sur le développenent er la commercialisation tourintigue du QUAD au Ouébec, Fédération des clubs de motoneigistes du Québec.

11 Zins Bcauchesne et associés (1996), Plans de marketing et de communication sw le produit vélo au Bas-Samt-Lauremt. ATR du BasSaint-Laurent, 26 mars.

12 Zins, Beauchesne et associes (1997), Eude des retombes et de l'impact économique de la Route verte du Bas-Sain-Laurent, Comité régional de la Route verte du Bas-SaintLaurent.

$13 \mathrm{Fid}$.

14 Zins Beauchesne et associes (1997), Diagnostic sectoriel de main-d'cuve de l'industrie dn towisme d'avenure, Société québécoise de developpenent de la main-d couve, aoutt. 